\title{
A New Approach to the Mode Theory of VLF Propagation
}

\author{
James R. Wait
}

(July 14, 1960)

\begin{abstract}
An attempt is made in this paper to present a concise derivation of the mode theory of VLF propagation. Taking note of the fact that the important modes for long-distance propagation are near grazing, suitable approximate forms of the wave functions are introduced at the outset, rather than at the end, of the analysis. It is thus possible to account for the influence of earth curvature in a relatively concise manner. The influence of the earth's magnetic field is also discussed. Finally, numerical results for the attenuation and the phase velocity of the dominant mode are presented.
\end{abstract}

\section{Introduction}

Despite the extensive work $[1-5]^{2}$ on the waveguide mode theory of VLF propagation, certain aspects of the subject are not yet resolved. The difficulty appears in finding suitable approximations to the spherical wave functions in the rigorous solution. It is the purpose of this paper to discuss this problem in some detail. For a background of the subject the reader is referred to a recent comprehensive paper on the general subject [6]. Here the solutions will be obtained from a somewhat different point of view.

\section{The Groundwave Field}

We will start by assuming that the source is a vertical electric dipole located on the surface of a smooth spherical earth of radius $a$, conductivity $\sigma$ and dielectric constant $\epsilon$. Spherical coordinates $(r, \theta, \phi)$ are chosen with the dipole located at $r=a$ and $\theta=0$. For harmonic time dependence the radial electric field component is written in the form

$$
E_{r} \cong \frac{e^{-i k a \theta}}{a(\theta \sin \theta)^{1 / 2}} V_{0} e^{i \omega \tau}
$$

apart from a constant factor. ${ }^{3}$ In the case of an airless earth, in which the ionosphere is neglected, it is well known that $V_{0}$ may be written in the form $[7,8]$

$$
V_{0} \cong 2(\pi x)^{1 / 2} e^{-i \pi / 4} \sum_{s=1,2, \ldots}^{\infty} \frac{e^{-i x t_{s}}}{\left(t_{s}-q^{2}\right)} \frac{w_{1}\left(t_{s}-y\right)}{w_{1}\left(t_{s}\right)},
$$

where

$$
x=\left(\frac{k a}{2}\right)^{1 / 3} \theta, y=\left(\frac{2}{k a}\right)^{1 / 3} k(r-a), i q=\left(\frac{k a}{2}\right)^{1 / 3} \Delta,
$$

1 Contribution from Central Radio Propagation Laboratory, National Bureau of Standards, Boulder, Colo.

${ }^{2}$ Figures in brackets indicate the literature references on page 46

${ }^{3}$ Equation (1) is normalized such that $V_{0}$ approaches 2 for $k a \rightarrow \infty$ and $\Delta \rightarrow 0$. and

$$
\Delta=\left(\frac{i \epsilon_{0} \omega}{\sigma+i \epsilon \omega}\right)^{1 / 2}\left[1-\frac{i \epsilon_{0} \omega}{\sigma+i \epsilon \omega}\right]^{1 / 2} .
$$

The coefficients $t_{s}$ are solutions of the equation

$$
w_{1}^{\prime}(t)-q w_{1}(t)=0,
$$

where $w_{1}(t)$ is an Airy integral and the prime indicates a derivative with respect to $t$. In terms of Hankel functions of order one third,

$$
w_{1}(t)=\exp (-2 \pi i / 3)(-\pi t / 3)^{1 / 2} H_{1 / 3}^{(2)}\left[(2 / 3)(-t)^{3 / 2}\right] .
$$

The above formula for $V_{0}$ is usually called the residue series representation for the groundwave field. It is often used as the basis for field strength calculations on a spherical smooth earth on the assumption that the ionosphere may be neglected or separately accounted for. It is valid when $k a>>1$ and $r-a<<a$. It is also required that the values of $\left|t_{s}\right|$ for the important modes are not large compared with unity. Such approximations as these are certainly valid for VLF waves.

For the purposes of the subsequent analysis $V_{0}$ is written as a contour integral in the manner

$$
V_{0}=e^{i \pi / 4}(x / \pi)^{\frac{1}{2}} \oint \frac{e^{-i x t} w_{1}(t-y)}{w_{1}^{\prime}(t)-q w_{1}(t)} d t .
$$

The contour encloses the poles at $t=t_{s}$ in a clockwise sense. The equivalence of eq (2) and (5) is easily verified on noting that

$$
w_{1}^{\prime \prime}\left(t_{s}\right)-q w_{1}^{\prime}\left(t_{s}\right)=\left(t_{s}-q^{2}\right) w_{1}\left(t_{s}\right)
$$

since

$$
w_{1}^{\prime \prime}(t)=t w_{1}(t),
$$

and

$$
w_{1}^{\prime}\left(t_{s}\right)=q w_{1}\left(t_{s}\right) .
$$




\section{The Sky Waves}

We will now enclose the earth by a concentric reflecting shell located at $r=a+h$ as indicated in figure 1. The electrical properties of this layer will not be specified at the moment, but it is assumed that an upgoing wave will be converted to a downgoing wave. Thus after one reflection

is converted to

$$
e^{-i x t} w_{1}(t-y)
$$

where

$$
A(t) e^{-i x t} w_{2}(t-y)
$$

$$
w_{2}(t)=\exp (2 \pi i / 3)(-\pi t / 3)^{1 / 2} H_{1 / 3}^{(1)}\left[(2 / 3)(-t)^{3 / 2}\right],
$$

and $A(t)$ is unknown function of $t$. The boundary condition at $r=a+h$ is written in the form

$$
\left[\frac{d w(t-y)}{d y}-q_{i} w(t-y)\right]_{y=y_{0}}=0
$$

where

$$
y_{0}=\left(\frac{2}{k a}\right)^{1 / 3} k h
$$

and $i q_{i}=\left(\frac{k a}{2}\right)^{1 / 3} \Delta_{i}$. The quantity $\Delta_{i}$ involves the properties of the layer beyond $r=a+h$; for the moment it is not given explicitly. Formally $\Delta_{i}=Z / \eta_{\circ}$ where $Z$ is the radial surface impedance at $r=a+h$. Most generally $\Delta_{i}$ (or $Z$ ) is a function of $t$ but usually it may be taken as a constant [6]. On identifying: $w(t-y)$ with $w_{1}(t-y)+A(t) w_{2}(t-y)$, it readily follows from eq (7) that

$$
A(t)=-\left[\frac{w_{1}^{\prime}\left(t-y_{0}\right)+q_{i} w_{1}\left(t-y_{0}\right)}{w_{2}^{\prime}\left(t-y_{0}\right)+q_{i} w_{2}\left(t-y_{0}\right)}\right] .
$$

The downcoming wave characterized by the function $w_{2}(t-y)$ is now reflected at the ground and thus it generates a new upgoing wave of the form

$$
A(t) B(t) w_{1}(t-y) e^{-i x t}
$$

The boundary condition at $r=a$ may be written

$$
\left[\frac{d w(t-y)}{d y}+q w(t-y)\right]_{y=0}=0
$$

Then on identifying $w(t-y)$ with the sum of the downcoming and the upgoing wave it follows that

$$
B(t)=-\left[\frac{w_{2}^{\prime}(t)-q w_{2}(t)}{w_{1}^{\prime}(t)-q w_{1}(t)}\right]
$$

The process may be repeated any number of times. The resultant field can thus be written as a sum

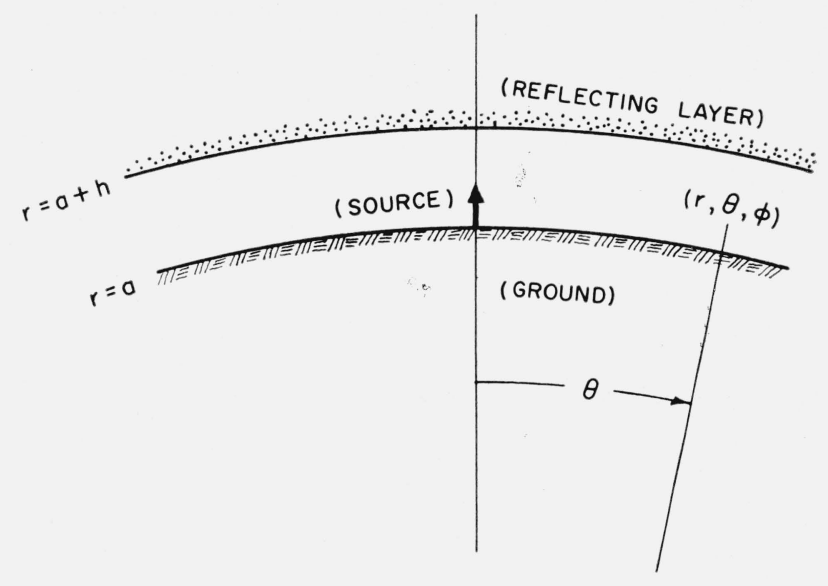

Figure 1. A sketch of the model employed in this paper.

The surface of the ground and the lower edge of the reflecting layer are concentric spherical surfaces.

$$
E_{1}=\frac{e^{-i k a \theta}}{a(\theta \sin \theta)^{1 / 2}} V
$$

where

$$
V=\sum_{j=0,1,2} V_{j}
$$

and

$$
V_{\mathrm{J}}=e^{i \pi / 4}\left(\frac{x}{\pi}\right)^{1 / 2} \oint \frac{e^{-i x t} w_{1}(t-y)}{w_{1}^{\prime}(t)-q w_{1}(t)}[A(t) B(t)]^{j / 2} d t
$$

for $j$ even, while

$$
V_{j}=e^{i \pi / 4}\left(\frac{x}{\pi}\right)^{1 / 2} \oint \frac{e^{-i x t} w_{2}(t-y)}{w_{1}^{\prime}(t)-q w_{1}(t)}[A(t)]^{\frac{j+1}{2}}[B(t)]^{\frac{j-1}{2}} d t
$$

for $j$ odd. Formally these are geometrical progressions so they can be summed. On interchanging the order of integration and summation, this leads to the integral representation

$$
\begin{aligned}
V & =e^{i \pi / 4}\left(\frac{x}{\pi}\right)^{1 / 2} \oint \frac{e^{-i x t}\left[w_{1}(t-y)+A(t) w_{2}(t-y)\right]}{\left[w_{1}^{\prime}(t)-q w_{1}(t)\right][1-A(t) B(t)]} d t \\
& =e^{i \pi / 4}\left(\frac{x}{\pi}\right)^{1 / 2} \oint \frac{e^{-i x t}\left[w_{1}(t-y)+A(t) w_{2}(t-y)\right]}{w_{1}^{\prime}(t)-q w_{1}(t)+A(t)\left\lfloor w_{2}^{\prime}(t)-q w_{2}(t)\right]} d t .
\end{aligned}
$$

Now the contour is to enclose the complex poles which occur at $t=t_{n}$ where $t_{n}$ is a solution of

$$
1-A(t) B(t)=0 .
$$

The residue series representation for the total field is thus given by 


$$
\begin{aligned}
& V=-2(\pi x)^{1 / 2} e^{-i \pi / 4} \\
& \underset{n=0,1,2}{\times} \sum_{\left[w_{1}\left(t_{n}\right)-q w_{1}\left(t_{n}\right)\right]\left[\frac{\partial}{\partial t} A(t) B(t)\right]} \frac{e^{-i x t_{n}}\left[w_{1}\left(t_{n}-y\right)+A\left(t_{n}\right) w_{2}\left(t_{n}-y\right)\right]}{t} .
\end{aligned}
$$

As in the residue series formula for the groundwave field, eq (17) is valid for $k a>>1$ and $h<<a$.

The interpretation of the preceding results is now discussed and certain simplifications made to facilitate computation. If $(-t)>>1$, it follows that

$$
\begin{aligned}
B(t) & =-\left[\frac{w_{2}^{\prime}(t)-q w_{2}(t)}{w_{1}^{\prime}(t)-q w_{1}(t)}\right] \\
& \simeq\left[\frac{-i(-t)^{1 / 2}-q}{i(-t)^{1 / 2}-q}\right] e^{-i \pi / 2} e^{i \frac{4}{3}(-t)^{3 / 2}} .
\end{aligned}
$$

On making the substitution $(-t)^{1 / 2}=\left(\frac{k a}{2}\right)^{1 / 3} C$ and noting that $i q=\left(\frac{k a}{2}\right)^{1 / 3} \Delta$, it follows that

$$
B(t) \cong \frac{C-\Delta}{C+\Delta} e^{i \pi / 2} e^{i \frac{4}{3}\left(\frac{k a}{2}\right) C^{3}} .
$$

If $C$ is identified as the cosine of an angle of incidence, $(C-\Delta) /(C+\Delta)$ can be recognized as a Fresnel reflection coefficient. Similarly, if $\left(y_{0}-t\right)>>1$

$$
A(t) \cong \frac{C^{\prime}-\Delta_{i}}{C^{\prime}+\Delta_{i}} e^{-i \pi / 2} e^{-i \frac{4}{3}\left(\frac{k a}{2}\right)\left(C^{\prime}\right)^{3}},
$$

where $\left(\frac{k a}{2}\right)^{1 / 3} C^{\prime}=\left(y_{0}-t\right)^{1 / 2} . \quad C^{\prime}$ may be identified as the cosine of the angle of incidence at the ionosphere. It is also noted that

$$
C^{\prime}=\left(C^{2}+\frac{2 h}{a}\right)^{1 / 2}
$$

The factor $\left(C^{\prime}-\Delta_{i}\right) /\left(C^{\prime}+\Delta_{i}\right)$ is, of course, a Fresnel reflection coefficient referred to the bottom of the layer at $r=a+h$.

The modal eq (16) which determines the coefficients $t_{n}$ may thus be written in the approximate form

where

$$
R_{g} R_{i} \exp (-i \hat{I})=1=e^{-i 2 \pi n},
$$

and

$$
R_{g}=\frac{C-\Delta}{C+\Delta} \text { and } R_{i}=\frac{C^{\prime}-\Delta_{i}}{C^{\prime}+\Delta_{i}}
$$

$$
\begin{aligned}
\hat{I} & =\frac{2 k a}{3}\left[\left(C^{\prime}\right)^{3}-C^{3}\right] \\
& =\frac{2 k a}{3}\left[\left(C^{2}+\frac{2 h}{a}\right)^{3 / 2}-C^{3}\right],
\end{aligned}
$$

if $\frac{h}{a}<<C^{2}$, then $R_{i}=\frac{C-\Delta_{i}}{C+\Delta_{i}}$ and $\hat{I} \cong 2 k h C$ which corresponds to the modal equation for the flat-earth case [5].

\section{Evaluation of the Residues}

For numerical work it is desirable to express the function

$$
\left[\frac{\partial}{\partial t} A(t) B(t)\right]_{t=t_{n}}
$$

explicitly in terms of Airy functions.

First, it is noted that

$$
\left[\frac{\partial}{\partial t} \frac{w_{2}^{\prime}(t)-q w_{2}(t)}{w_{1}^{\prime}(t)-q w_{1}(t)}\right]_{t=t_{n}}=\frac{-2 i\left(t_{n}-q^{2}\right)}{\left[w_{1}^{\prime}\left(t_{n}\right)-q w_{1}\left(t_{n}\right)\right]^{2}},
$$

where use has been made of the relations

(1) $w_{1}^{\prime \prime}(t)=t w_{1}(t)$ and

(2) $w_{1}(t) w_{2}^{\prime}(t)-w_{1}^{\prime}(t) w_{2}(t)=-2 i$ which are valid for any value of $t$. Similarly,

$$
\begin{aligned}
{\left[\frac{\partial}{\partial t} \frac{w_{1}^{\prime}\left(t-y_{0}\right)+q_{i} w_{1}\left(t-y_{0}\right)}{w_{2}^{\prime}\left(t-y_{0}\right)+q_{i} w_{2}\left(t-y_{0}\right)}\right]_{t=t_{n}} } \\
=\frac{2 i\left(t_{n}-y_{0}-q_{i}^{2}\right)}{\left[w_{2}^{\prime}\left(t_{n}-y_{0}\right)+q_{i} w_{2}\left(t_{n}-y_{0}\right]^{2}\right.} .
\end{aligned}
$$

Thus

$$
\begin{aligned}
& {\left[\frac{\partial}{\partial t} A(t) B(t)\right]_{t=t_{n}}} \\
& \quad=\frac{-2 i\left(t_{n}-q^{2}\right)}{\left[w_{1}^{\prime}\left(t_{n}\right)-q w_{1}\left(t_{n}\right)\right]\left[w_{2}^{\prime}\left(t_{n}\right)-q w_{2}\left(t_{n}\right)\right]} \\
& \quad+\frac{2 i\left(t_{n}-y_{0}-q_{i}^{2}\right)\left[w_{2}^{\prime}\left(t_{n}\right)-q w_{2}\left(t_{n}\right)\right]}{\left.w_{1}^{\prime}\left(t_{n}\right)-q w_{1}\left(t_{n}\right)\right]\left[w_{2}^{\prime}\left(t_{n}-y_{0}\right)+q_{i} w_{2}\left(t_{n}-y_{0}\right)\right]^{2}} .
\end{aligned}
$$

Therefore, the complete residue series may be written

$$
\begin{gathered}
V=(\pi x)^{1 / 2} e^{-i 3 \pi / 4} \\
\underset{n=0,1,2}{\times} \frac{e^{-i x t}{ }_{n}\left[w_{1}\left(t_{n}-y\right)+A\left(t_{n}\right) w_{2}\left(t_{n}-y\right)\right]}{\left.\frac{t_{n}-q^{2}}{w_{2}^{\prime}\left(t_{n}\right)-q w_{2}\left(t_{n}\right)}-\frac{\left(t_{n}-y_{0}-q_{i}^{2}\right)\left[w_{2}^{\prime}\left(t_{n}\right)-q w_{2}\left(t_{n}\right)\right]}{\left[w_{2}^{\prime}\left(t_{n}-y_{0}\right)+q_{i} w_{2}\left(t_{n}-y_{0}\right)\right]^{2}}\right]} .
\end{gathered}
$$

Numerical evaluation of the series is straightforward if tables of the Airy functions of complex argument are used. In the situation where

$$
\left(-t_{n}\right)^{1 / 2}=\left(\frac{k a}{2}\right)^{1 / 3} C_{n} \gg 1
$$

the Airy functions may be replaced by the first term of their asymptotic expansions. Under this approximation and after considerable algebraic reduction, it is possible to write, for $y=0$,

$$
V=4 e^{-i \pi / 4}(2 \pi k a \theta)^{1 / 2} \sum_{n=0,1,2} \ldots\left[\frac{e^{i k a \theta C_{n}^{2} / 2} F_{n}}{\left.\frac{\partial \hat{I}}{\partial C}+\frac{i \frac{\partial}{\partial C} R_{g} R_{i}}{R_{g} R_{i}}\right]_{C=C_{n}}}\right.
$$


where

$$
R_{g}=\frac{C-\Delta}{C+\Delta}, \quad R_{i}=\frac{C^{\prime}-\Delta_{i}}{C^{\prime}+\Delta_{i}}, \quad F_{n}=\frac{C_{n}^{2}}{C_{n}^{2}-\Delta^{2}}
$$

and

$$
\hat{I}=\frac{2 k a}{3}\left[\left(C^{\prime}\right)^{3}-C^{3}\right] .
$$

If the height of the source dipole is $z_{0}=r_{0}-a$ and the observer is $z=r-a$, the $F_{n}$ in the above expression should be replaced by

where

$$
F_{n}=f_{n}(z) f_{n}\left(z_{0}\right)
$$

$$
\begin{aligned}
2 f_{n}(z)= & R_{g}{ }^{-1 / 2} \exp \left[i k \int_{0}^{z}\left(C_{n}^{2}+\frac{2 z}{a}\right)^{1 / 2} d z\right] \\
& +R_{g}{ }^{1 / 2} \exp \left[-i k \int_{0}^{z}\left(C_{n}^{2}+\frac{2 z}{a}\right)^{1 / 2} d z\right] \\
= & R_{g}{ }^{-1 / 2} \exp \left\{\frac{i k a}{3}\left[\left(C_{n}^{2}+\frac{2 z}{a}\right)^{3 / 2}-C_{n}^{3}\right]\right\} \\
& +R_{g}{ }^{1 / 2} \exp \left\{\frac{i k a}{-3}\left[\left(C_{n}^{2}+\frac{2 z}{a}\right)^{3 / 2}-C_{n}^{3}\right]\right\} .
\end{aligned}
$$

If $2 z / a<<C_{n}^{2}$ it is permissible to use

$$
2 f_{n}(z) \cong R_{g}{ }^{-1 / 2} \exp \left(i k C_{n} z\right)+R_{g}{ }^{1 / 2} \exp \left(-i k C_{n} z\right)
$$

and if $R_{g} \cong 1$ corresponding to a perfectly conducting ground,

$$
f_{n}(z) \cong \cos k C_{n} z .
$$

The value of $C_{n}$ to employ in the residue series is related, of course, to $t_{n}$ by

$$
C_{n}=\left(\frac{2}{k a}\right)^{1 / 3}\left(-t_{n}\right)^{1 / 2} .
$$

Under the condition that $\left(-t_{n}\right)^{1 / 2}>>1$, the rootdetermining equation becomes

$$
R_{i} R_{g} e^{-i(2 k h+V) C_{n}}=e^{-2 \pi i n}
$$

where $V$ is defined by

$$
(2 k h+V) C_{n}=\frac{2 k a}{3}\left[\left(C_{n}^{\prime}\right)^{3}-C_{n}^{3}\right]=\hat{I}_{n} .
$$

Noting that

$$
\begin{aligned}
\frac{\partial \hat{I}_{n}}{\partial C_{n}} & =2 k a\left[\left(C_{2}^{n}+\frac{2 h}{a}\right)^{1 / 2}-C_{n}^{2}\right] \\
& \simeq 2 k h \text { if } 2 h / a \ll C_{n}^{2} \\
& \simeq 2 k(2 a h)^{1 / 2} \text { if } 2 h / a \gg C_{n}^{2},
\end{aligned}
$$

it is seen that the second term within the square bracket on the right-hand side is small if $k h$ is reasonably large and if the losses are small.

\section{Discussion of Various Approximations}

As a check on the algebra it may be noted that under the flat-earth approximation (i.e., $2 h / a<<C_{n}^{2}$ ), the residue series formula may be written

$$
V=\frac{2(\rho / \lambda)^{1 / 2}}{(h / \lambda)} e^{i}\left(\frac{2 \pi \rho}{\lambda}-\frac{\pi}{4}\right) \sum_{n=0,1,2 \ldots} \delta_{n} e^{-i 2 \pi \rho S_{n} / \lambda} f_{n}(z) f_{n}\left(z_{0}\right),
$$

where

$$
\delta_{n}=\left[1+i \frac{\partial\left[R_{i} R_{g}\right] / \partial C}{2 k h R_{i} R_{g}}\right]_{C=C n}^{-1},
$$

$\rho=a \theta$, and $S_{n}=\left(1-C_{n}{ }^{2}\right)^{1 / 2} \cong 1-C_{n}^{2} / 2$. For practical purposes, $\delta_{0} \cong 1 / 2$ and $\delta_{n} \cong 1$ for $n=1,2,3 \ldots$. Equation (32) agrees with an earlier analysis $[5,6]$.

For a first approximation it is probably permissible to neglect the curvature altogether. To improve on this it is important to retain curvature dependent terms in a consistent manner. For example, at VLF, the restriction $C_{n}^{2} \gg 2 h / a$ is not as stringent as

$$
C_{n} \gg(2 / k a)^{1 / 3} .
$$

Thus eq (27) for $V$ is probably not any better (if as good) as eq (32). To effect a real improvement one should retain the Airy functions of argument $t_{n}$ without approximation, but allow the Airy functions of argument $t_{n}-y_{0}$ to be replaced by the first terms of their asymptotic expansions. This is justified since $y_{0}$ is reasonably large compared to unity in the VLF range. With this approach the root-determining equation may be written

$$
R_{i} R_{g} F_{g} e^{-i(2 k h+V) C}=e^{-2 \pi i n}=1
$$

where

$$
F_{g}=\frac{\frac{w_{2}^{\prime}(t)-q w_{2}(t)}{w_{1}^{\prime}(t)-q w_{1}(t)}}{i \exp \left[i \frac{4}{3}(-t)^{3 / 2}\right]\left[\frac{-i(-t)^{1 / 2}-q}{i(-t)^{1 / 2}-q}\right]} .
$$

If the ground is assumed to be perfectly conducting $R_{g}=1$ and $q=0$. Then, on writing

$$
R_{i}=-e^{-2 \beta C^{\prime}}
$$

the mode equation reduces to

$$
2 \pi n=\pi-2 i \beta C^{\prime}+\left[\left(C^{\prime}\right)^{3}-C^{3}\right] \frac{2}{3} k a+i \arg F_{g},
$$

where now

$$
F_{g}=\frac{w_{2}^{\prime}(t)}{w_{1}^{\prime}(t)} \exp \left[i \frac{\pi}{2}-i \frac{4}{3}(-t)^{3 / 2}\right] .
$$

For long-distance propagation in the VLF range, only the mode corresponding to $n=1$ is of importance. 
Furthermore, since $C$ is small, for a first approximation

$$
\frac{w_{2}^{\prime}(t)}{w_{1}^{\prime}(t)} \cong e^{i \pi / 6}
$$

also, $\beta$ is essentially a constant and is approximately equal to $1 / \Delta_{i}$. Thus

$$
C^{2} \cong \frac{\frac{7 \pi}{6}-\frac{2}{3}(k a)\left(\frac{2 h}{a}\right)^{3 / 2}+2 i \beta\left(\frac{2 h}{a}\right)^{1 / 2}}{k a\left(\frac{2 h}{a}\right)^{1 / 2}-i \beta\left(\frac{a}{2 h}\right)^{1 / 2}}
$$

This formula should be a fairly good approximation if $\left|C^{2}\right|$ is somewhat less than $2 h / a$. For example, if $\beta=0$, corresponding to $R_{i}=-1$, and if $h / a=10^{-2}$ the above may be written

$$
C^{2}=\frac{0.041248}{(h / \lambda)}-0.01334
$$

For $h / \lambda$ in the region from 2.5 to $5,\left|C^{2}\right|$ is never greater than $5 \times 10^{-3}$.

Using perturbation methods, higher order approximations for the solution of eq (35) may be obtained. Details of such computations will not be described here.

\section{Certain Extensions of the Theory}

The preceding analysis has referred specifically to a vertical electric dipole. If the source is a vertical magnetic dipole the results derived above are still applicable if the reflection coefficients $R_{g}$ and $R_{i}$ are replaced by their counterparts $R_{g}^{h}$ and $R_{i}^{h}$ for horizontal polarization. The only other change is that $q$ is now defined by

$$
i q=\left(\frac{k a}{2}\right)^{1 / 3} \frac{1}{\Delta_{h}} \quad \text { where } \quad \frac{1}{\Delta_{h}}=\left[\frac{\sigma+i \epsilon \omega}{i \epsilon_{0} \omega}-1\right]^{1 / 2} .
$$

Since $[q] \gg 1$, under all practical conditions,

$$
F_{g}^{h} \cong \frac{w_{2}(t)}{w_{1}(t)} \exp \left[-i\left(\frac{\pi}{2}+\frac{4}{3}(-t)^{3 / 2}\right)\right],
$$

which is to be used instead of $F_{g}$. The modes excited by a magnetic dipole are of a TE (transverse electric) type whereas for an electric dipole they are of a TM (transverse magnetic) type.

\section{Application to Specific Ionospheric Models}

In the case of a homogeneous isotropic ionosphere with a sharp lower boundary, the Fresnel reflection coefficient for small $C^{\prime}$ may be written

$$
R_{i}=\frac{C^{\prime}-\Delta_{i}}{C^{\prime}+\Delta_{i}} \cong-e^{-2 \beta C^{\prime}},
$$

where $\beta \cong \frac{1}{\Delta_{i}}$. In terms of the plasma frequency $\omega_{0}$ and the collision frequency $\nu$

$$
\beta \cong\left(1-i \omega_{r} / \omega\right)\left(-i \omega_{r} / \omega\right)^{-1 / 2}
$$

where $\omega_{r}=\omega_{0}^{2} / \nu$ under the condition that $\omega \ll \nu$.

Unfortunately, the presence of the earth's magnetic fields renders the ionosphere anisotropic. The reflection coefficient is thus a matrix of the form [1]

$$
\left[R_{i}\right]=\left[\begin{array}{cc}
{ }_{\|} R_{\|} & { }_{\|} R_{\perp} \\
{ }_{\perp} R_{\|} & { }_{\perp} R_{\perp}
\end{array}\right],
$$

where the coefficients ${ }_{\|} R_{\|},{ }_{\|} R_{\perp},{ }_{\perp} R_{\|}$, and ${ }_{\perp} R_{\perp}$ indicate the complex ratio of a specified electric field in the wave after reflection to a specified electric field in the wave before reflection. The first subscript denotes whether the electric field in the incident wave is parallel $(\|)$ or perpendicular $\left(_{\perp}\right)_{\text {) }}$ to the plane of incidence and the second subscript refers in the same way to the reflected wave. It is understood, of course, that $\left[R_{i}\right]$ is a function of $C^{\prime}$, the cosine of the angle of incidence at the ionosphere. Following an earlier analysis [6], the generalization of eq (33), to include the influence of anisotropy, may be expressed in the form

$$
\begin{aligned}
& {\left[1-{ }_{\|} R_{\|} R_{g} F_{g} e^{-i(2 k h+V) C}\right] } \\
\times & {\left[1-{ }_{\perp} R_{\perp} R_{g}^{h} F_{g}^{h} e^{-i(2 k h+V) C}\right] } \\
- & R_{\perp \perp} R_{\|} R_{g} F_{g} R_{g}^{h} F_{g}^{h} e^{-i 2(2 k h+V) C}=0,
\end{aligned}
$$

where $R_{g}, R_{g}^{h}, F_{g}, F_{g}^{h}$, and $V$ have been defined. Strictly speaking, equation (40) is only valid when there is perfect azimuthal symmetry about the source dipole.

It may be noted that if the conversion coefficients ${ }_{\|} R_{\perp}$ and ${ }_{\perp} R_{\|}$vanish, the modal equation splits into two parts which are

$$
1-{ }_{\|} R_{\|} R_{g} F_{g} e^{-i(2 k h+V) C}=0
$$

and

$$
1-{ }_{\perp} R_{\perp} R_{g}^{h} F_{g}^{h} e^{-i(2 k h+V) C}=0 .
$$

These two uncoupled equations give the solutions for the TM and TE modes, respectively. In the more general case the modes are coupled. However, for low-order modes and particularly for the dominant mode $(n=1)$, the coupling is extremely small and a good first-order approximation is obtained on the assumption that coupling may be neglected. Furthermore, the TE mode is only of academic or secondary interest since it is not excited to any extent by a vertical antenna. Thus the constants of the first-order mode may be obtained from eq (37) with an appropriate value of $\beta$. 
For propagation in the magnetic meridian or for propagation in polar regions the QL (quasi-longitudinal) approximation [9] to describe conditions within the ionosphere is valid. The refractive index $\mu$, in this case, is given by [1]

$$
\mu^{2}=1-i\left(\omega_{r} / \omega\right) \exp \left( \pm i \phi_{L}\right)
$$

where

$\tan \phi_{L}=\frac{\omega_{L}}{\nu}=\frac{\text { longitudinal component of gyrofrequency }}{\text { collision frequency }}$

and $\omega_{r}=\omega_{0}^{2}\left(\nu^{2}+\omega_{L}^{2}\right)^{-1 / 2}$. Thus for highly oblique incidence (i.e., $C$ is small) it may be shown that [6]

$$
R \cong-e^{-2 \beta C^{\prime}}
$$

where

$$
\beta=\frac{\mu_{0} \mu_{e}}{\mu_{0}+\mu_{e}} \frac{C_{0}+C_{e}}{C_{0} C_{e}}
$$

where $\mu_{0}$ and $\mu_{e}$ denote the two values of $\mu$, and

$$
C_{0}=\left(1-1 / \mu_{0}^{2}\right)^{\frac{1}{2}} \text { and } C_{e}=\left(1-1 / \mu_{e}^{2}\right)^{\frac{1}{2}} .
$$

The QL approximation mentioned above is only valid when [10]

$$
\frac{\omega_{T}^{4}}{4 \omega^{2} \omega_{L}^{2}}<<\left|\left(1-\frac{\omega_{0}^{2}}{\omega^{2}}-\frac{\nu}{\omega}\right)^{2}\right|
$$

where $\omega_{L}$ and $\omega_{T}$ are the longitudinal and transverse components of the (angular) gyrofrequency. As mentioned above, this condition is satisfied for propagation in the magnetic meridian and/or for a steeply dipping field. Clearly, this condition is violated when the transverse component of the earth's magnetic field is large such as for propagation around the magnetic equator. The case of a purely horizontal and transverse field has been considered by Barber and Crombie [11]. Using their results, which are applicable to a sharply bounded ionosphere, it may be readily shown that [6]

$$
{ }_{\|} R_{\|} \cong-e^{-2 \beta C^{\prime}}, \quad{ }_{\|} R_{\perp}=0
$$

where now

$$
\beta=\frac{(1+i L)^{2}-\gamma^{2}}{(1+i L)^{1 / 2}\left(i L-L^{2}-\gamma^{2}\right)^{1 / 2}-i \gamma}
$$

and

$$
L=\frac{\omega}{\omega_{r}}=\frac{\omega \nu}{\omega_{0}^{2}} \text { and } \gamma=\frac{\omega_{T} \omega}{\omega_{0}^{2}} .
$$

For east-to-west propagation, $\gamma$ is positive, while for west-to-east propagation, $\gamma$ is negative. In the present situation it is convenient to define an angle
$\phi_{T}$ such that

$$
\begin{aligned}
& \gamma=+L\left|\tan \phi_{T}\right| \text { for east-to-west propagation } \\
& =-L\left|\tan \phi_{T}\right| \text { for west-to-east propagation. }
\end{aligned}
$$

For propagation at any angle with respect to the earth's magnetic field and for an arbitrary angle of dip $I$, it is necessary to seek more general solutions. First, one must solve a quartic equation for the refractive indices (ordinary and extraordinary components). Then on matching tangential field components at the air-ionosphere boundary, an explicit expression for the reflection coefficient may be obtained [12]. Using this quite general approach Johler and Walters [13] have obtained extensive numerical results for the coefficients ${ }_{\| \mid} R_{\|,\|} R_{\perp},{ }_{\perp} R_{\perp}$, and ${ }_{\perp} R_{\|}$for various (real) angles of incidence and range of values for the electron density, $N$, collision frequency $\nu$, dip-angle $I$ and the azimuthal direction of the wave $\phi_{a}$ (measured clockwise from the north). Formally, the expressions for these reflection and conversion coefficients may be substituted in eq (13) which, in principle, can be solved for the modes in the general case. For VLF and at highly oblique angles of incidence (e.g., $82^{\circ}$ ), the quantity $\left.\right|_{\|} R_{\perp} \times$ ${ }_{\perp} R_{\|} \mid$is of the order of $10^{-3}$ or $10^{-4}$ and thus coupling between the TE and TM modes is negligible. At least this is true for the range of values of the ionospheric parameters considered here. Thus the uncoupled modal equation may again be used for an adequate first approximation after having determined the appropriate value of $\beta$.

\section{Presentation of Results}

Using the above approaches numerical results have been obtained for the complex values of $C$ for the dominant mode. ${ }^{4}$ From this quantity, it is a simple matter to compute the corresponding value of $S$ which describes the propagation in the horizontal direction. For example, the attenuation per unit distance is proportional to the quantity $-H \operatorname{Im} S$ and the phase velocity relative to $c$ is $1 / \operatorname{Re} S$. In the present paper, these are the quantities of prime interest since they are adequate to describe the propagation characteristics of VLF radio waves at large distances from the source (i.e., $>2,000 \mathrm{~km}$ ). At shorter distances higher order modes should be considered. Their influence has been discussed elsewhere [5].

In figure 2 the attenuation coefficient, for the dominant mode $(n=1)$, is plotted as a function of $H(=h / \lambda)$ for a curved earth where $h / a=0.01$. The upper- and right-hand scales in this figure and in many which follow, are a frequency scale and attenuation scale in decibels per $1,000 \mathrm{~km}$ of path length applicable to a height of $70 \mathrm{~km}$. The quasilongitudinal approximation is employed and $\phi_{L}$ is either $60^{\circ}$ or $0^{\circ}$; the latter, of course, corresponds to no magnetic field. The constant $B$ on the curves is

${ }^{4}$ For sake of brevity, the subscript 1, to indicate the first mode, is dropped in what follows. 
defined by

$$
B=\frac{L}{H} \text { where } \frac{1}{L}=\frac{\omega_{r}}{\omega}=\frac{\omega_{0}^{2}}{\omega\left(\nu^{2}+\omega_{L}^{2}\right)^{1 / 2}},
$$

or alternately

$$
B=\frac{2 \pi c}{\omega_{r} h} .
$$

FREQUENCY, $k c / s$

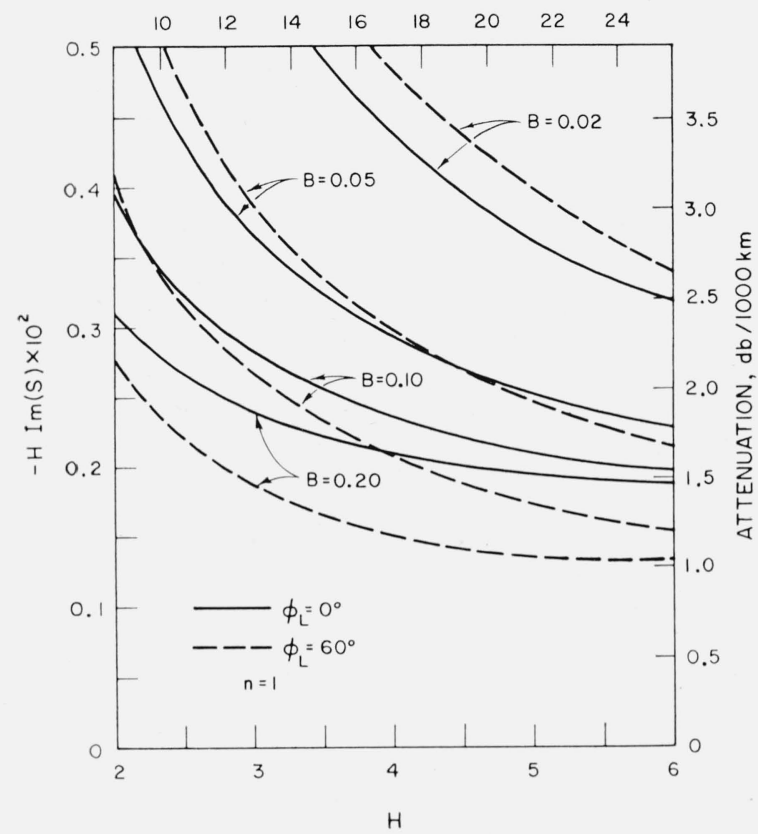

Figure 2. Attenuation coefficient,- $\mathrm{H}$ Im $\mathrm{S}$, as a function of $\mathrm{H}(=\mathrm{h} / \lambda)$ for the dominant mode.

The upper and the right-hand scales are for $h=70 \mathrm{~km}$.

The four $B$ values on the curves $(0.02,0.05,0.1$, and 0.2 ) correspond to $\omega_{r}$ values of $13,5.4,2.7$, and $1.3 \times 10^{5}$, respectively. For a given value of $B$, it is seen that the attentuation depends on $\phi_{L}$. For large values of $B$ (i.e., poorly conducting ionosphere) the influence of the terrestrial magnetic field is to lower the attenuation whereas the converse is true at smaller values of $B$. Since $B$ is in the range 0.05 to 0.01 typically for daytime conditions, the influence of the magnetic field is small under the quasilongitudinal approximation.

The relative phase velocity of the dominant mode is shown in figures $3 \mathrm{a}$ and $3 \mathrm{~b}$ for $\phi_{L}=0$ and $60^{\circ}$, respectively. The (upper) frequency scale is for a height of $70 \mathrm{~km}$. From these it appears that the influence of the magnetic field is quite small. It is rather interesting to note that for a frequency of $14 \mathrm{kc} / \mathrm{s}$ the phase velocity is very near, $c$, the velocity of light. ${ }^{5}$ It should be remembered, of course, that the phase velocity is referred to the surface of the ground in this particular analysis.

Since one is interested in relatively small deviations of $1 / \operatorname{Re}(S)$ from unity, considerable care is needed in approximating the wavefunctions involved. The results shown in figures $3 \mathrm{a}$ and $3 \mathrm{~b}$ for a curved earth

${ }_{5}^{5}$ Recent experimental data appear to confirm this point (M. L. Tibbals, private communication). are not expected to be accurate to more than a few parts in $10^{4}$.

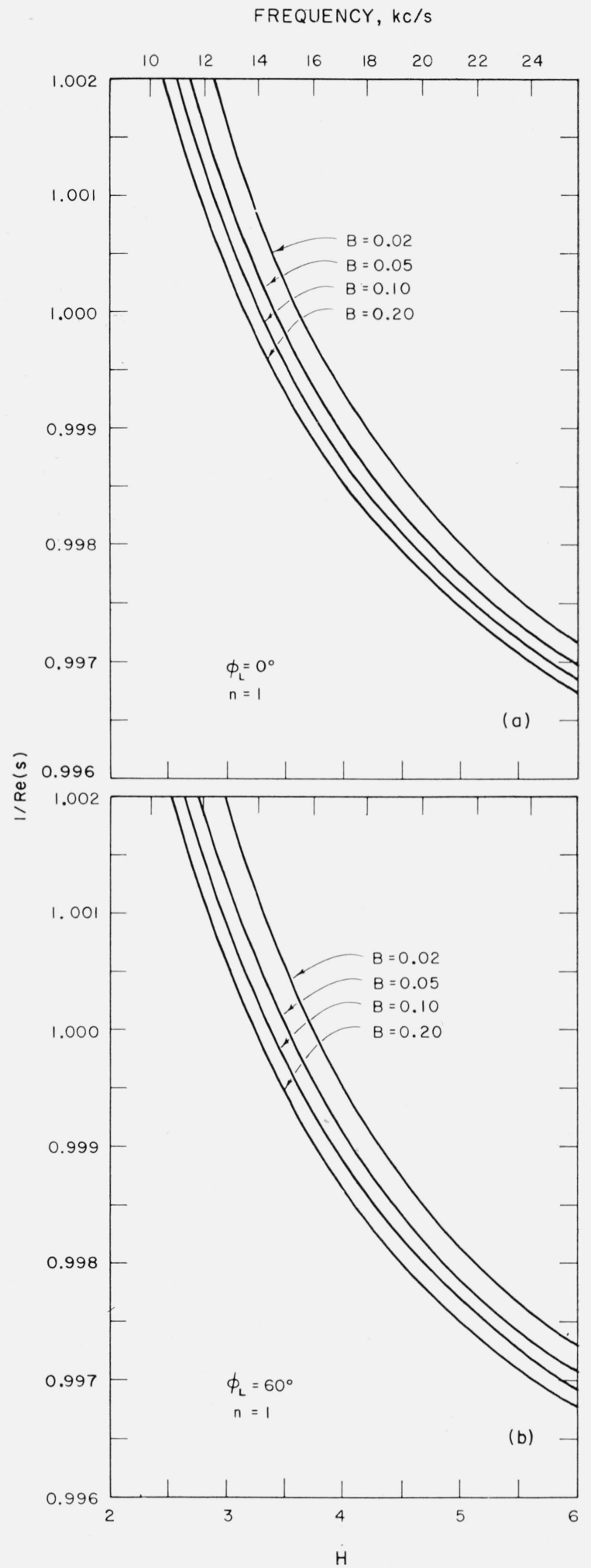

Figures $3 \mathrm{a}$ and $\mathrm{b}$. The relative phase velocity, $1 /$ Re $\mathrm{S}$ as:a function of $\mathrm{H}$ for the dominant mode.

The upper-frequency scale is for $h=70 \mathrm{~km}$. 
The calculation of the mode characteristics for a magnetic field of arbitrary direction is, of course, quite involved. However, approximate results can be obtained if coupling between TE and TM type modes is neglected [6]. Actually, in the case of a purely transverse field, the coupling is zero anyway.

In the following the influence of an arbitary magnetic field is demonstrated by calculating the ratio, $P$, of the attenuation with a magnetic field to the attenuation without the magnetic field. Various values of the dip-angle $I$, and the azimuthal direction of propagation, $\phi_{a}$, are chosen. The ratio, $Q$, of the phase-velocity deviations with and without a magnetic field is also considered. Specifically, these ratios are

$P=\frac{\text { Attenuation with magnetic field }}{\text { Attenuation without magnetic field }}=\frac{\operatorname{Im} S}{[\operatorname{Im} S]_{H=0}}$

and

$Q=\frac{\text { Phase velocity deviation with magnetic field }}{\text { Phase velocity deviation without magnetic field }}$

$$
=\frac{\left[\frac{1}{\operatorname{Re} S}-J\right]}{\left[\frac{1}{\operatorname{Re} S}-J\right]_{H=0}} \text { where } J \cong \frac{2 a}{2 a+h}
$$

To obtain numerical values of $P$ and $Q$, computed data from J. R. Johler [14] were requested for the reflection coefficient ${ }_{\|} R_{\|}$for a real angle of incidence of $82^{\circ}$ and at frequencies of $10,12,14,16,20$, and $22 \mathrm{kc} / \mathrm{s}$. The appropriate value of $\beta$ was then calculated from

$$
{ }_{\|} R_{\|}=-e^{-2 \beta C^{\prime}},
$$

where $C^{\prime}=\cos 82^{\circ}$. The electron density values for the sharply bounded model were $10^{3}$ and $3 \times 10^{3}$ electrons $/ \mathrm{cm}^{3}$, respectively. The collision frequency was taken to be $2 \times 10^{7}$ and the strength of the earth's magnetic field was 0.5 gauss. The angle $\phi_{a}$ which defined the direction of propagation measured clockwise from north assumed the values $0^{\circ}, 60^{\circ}, 120^{\circ}, 180^{\circ}, 240^{\circ}$, and $300^{\circ}$. As a check on the method, values of $\beta$ were calculated directly from eq (40) which is applicable to $\phi_{a}=90^{\circ}$ and $180^{\circ}$ for $I=0$.

The results for the attenuation ratios are shown in figures $4 \mathrm{a}$ and $4 \mathrm{~b}$ for a dip-angle $I$ of $0^{\circ}$, in figures $5 \mathrm{a}$ and $5 \mathrm{~b}$ for an $I$ of $45^{\circ}$, and in figures $6 \mathrm{a}$ and $6 \mathrm{~b}$ for an $I$ of $84.3^{\circ}$.

These results would indicate that nonreciprocity is most evident in propagation around the geomagnetic equator. North-to-south or south-to-north propagation appears to be almost reciprocal. It is also evident that for this range of electron densities, the nonreciprocity for attenuation is more marked for the smaller densities. It is also apparent from these results that the nonreciprocal effects are

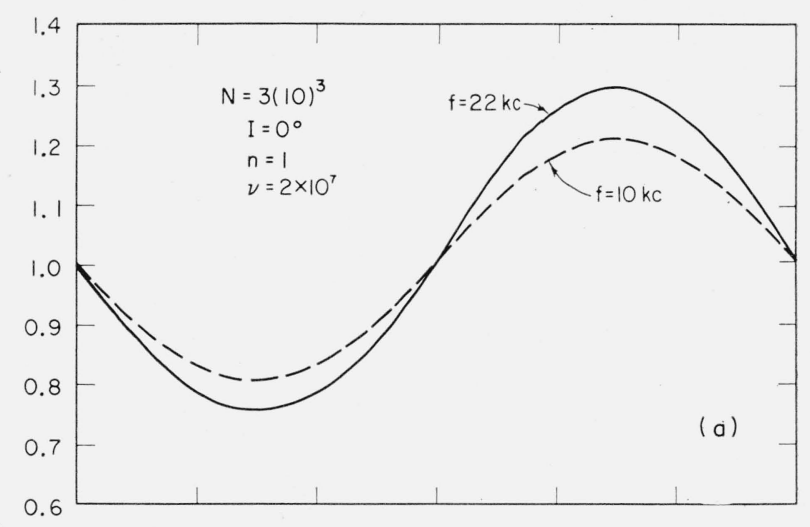

$P$

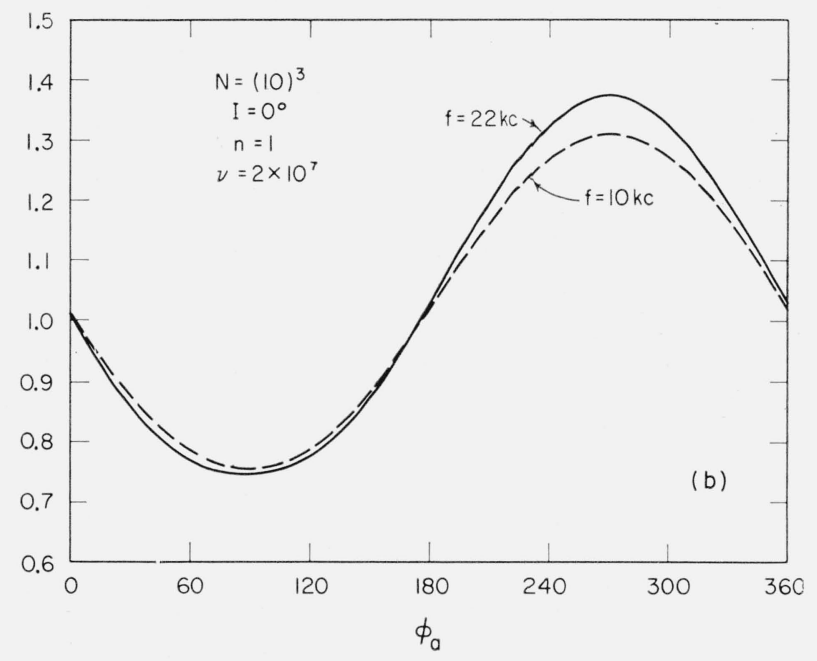

Figures $4 \mathrm{a}$ and $\mathrm{b}$. The ratio of the attenuation rates with and without a (transverse) terrestrial magnetic field. $\phi_{a}$ is the direction of propagation with respect to geomagnetic north.

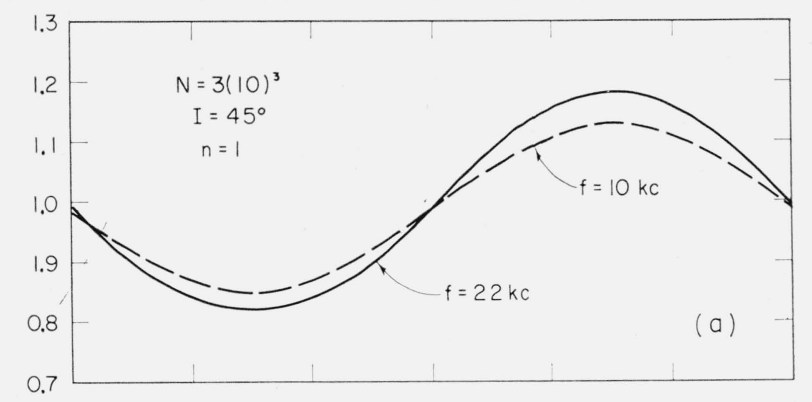

$\mathrm{P}$

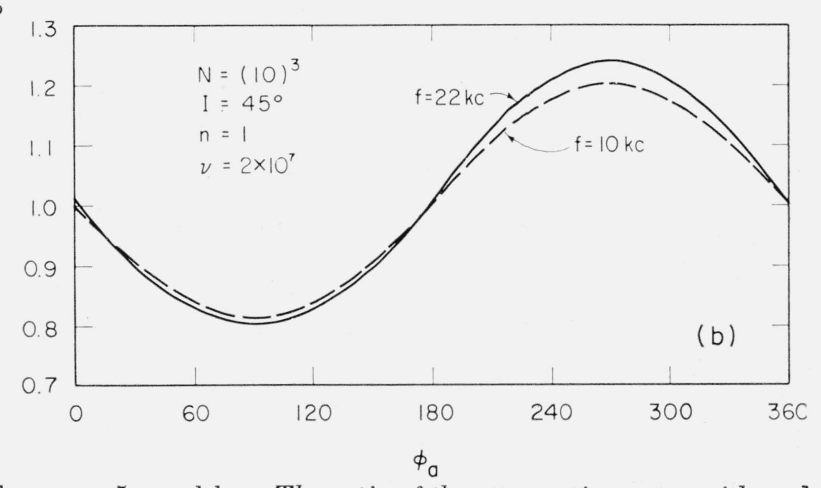

Figures $5 \mathrm{a}$ and $\mathrm{b}$. The ratio of the attenuation rates with and without a terrestrial magnetic fueld for a dip angle of $45^{\circ}$. 
diminished as the magnetic field approaches the vertical. Qualitatively the curves are very similar and there is a strong suggestion that nonreciprocity is proportional to the component of the earth's magnetic field which is horizontal and transverse to the direction of propagation.
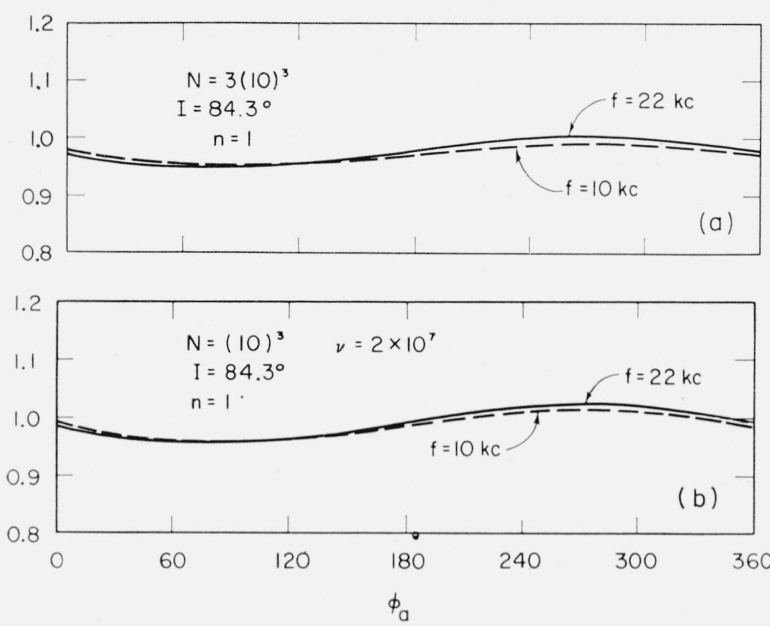

Figures $6 \mathrm{a}$ and $\mathrm{b}$. The ratio of the aitenuation rates with and without a terrestrial magnetic field for a dip angle of $84.3^{\circ}$.
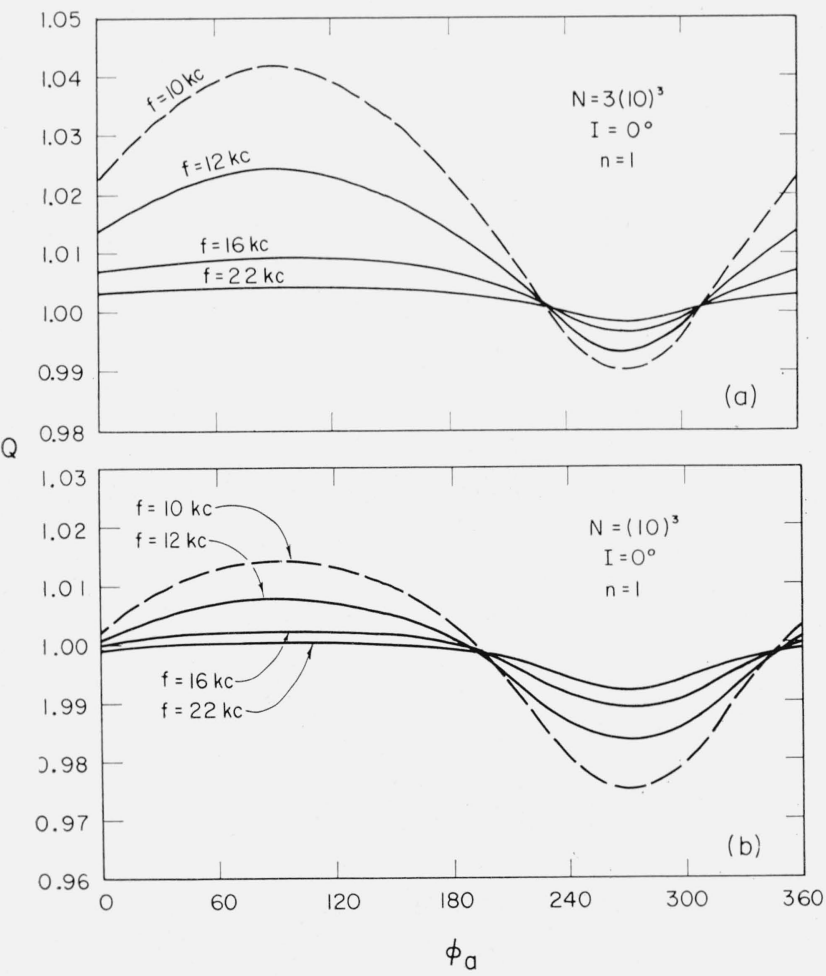

Figures $7 \mathrm{a}$ and $\mathrm{b}$. The ratio of the phase-velocity deviations with and without a (transverse) terrestrial magnetic field.

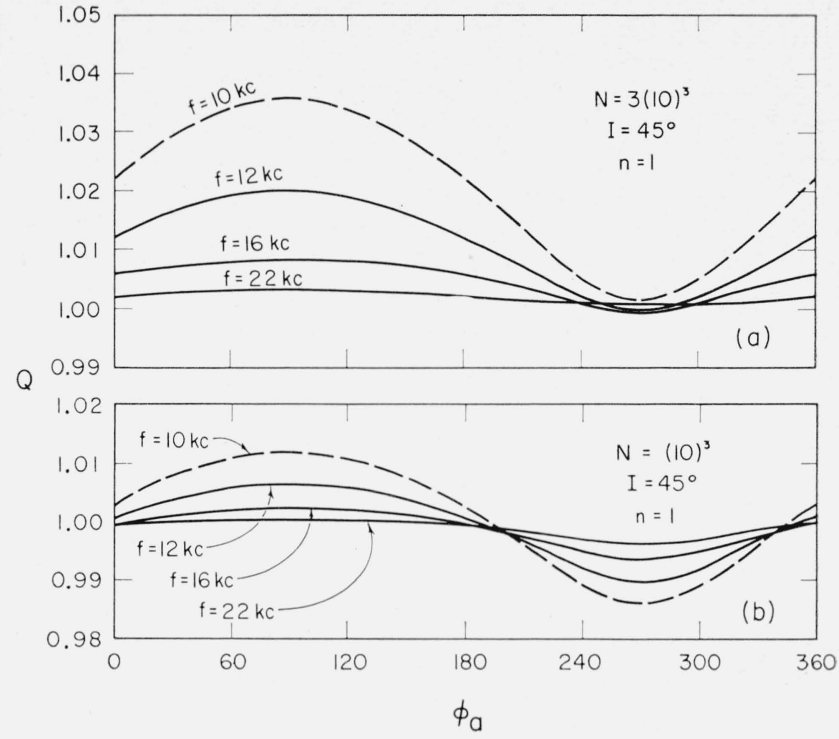

Figures $8 \mathrm{a}$ and $\mathrm{b}$. The ratio of the phase-velocity deviations with and without a terrestrial magnetic field for a dip angle of $45^{\circ}$.

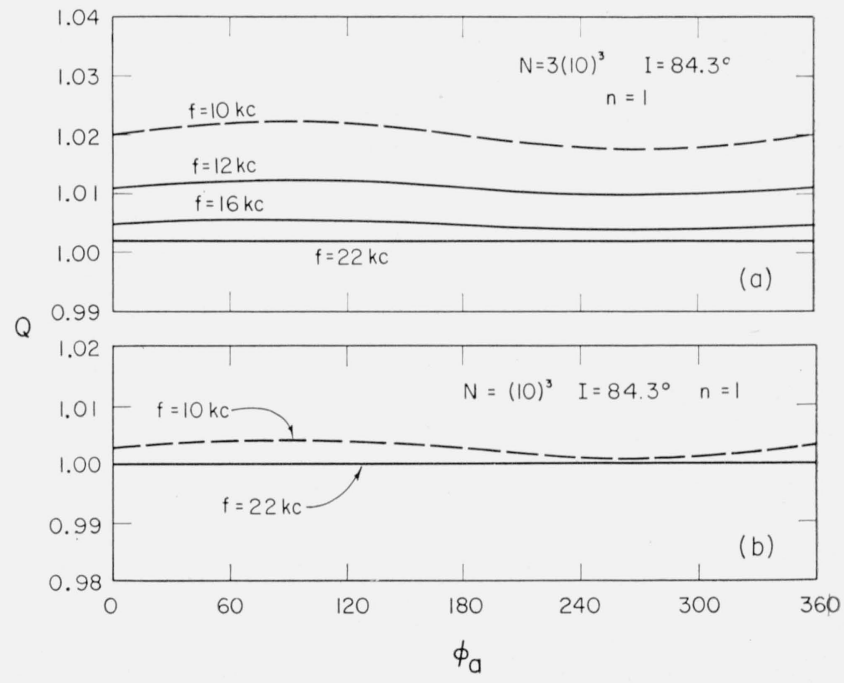

Figures $9 \mathrm{a}$ and $\mathrm{b}$. The ratio of the phase-velocity deviations with and without a terrestival magnetic field for a dip angle of $84.3^{\circ}$.

The corresponding curves for the ratio $Q$ of the phase velocity deviations are shown in figures $7 \mathrm{a}$ and $7 \mathrm{~b}$ for $I=0$, in figures $8 \mathrm{a}$ and $8 \mathrm{~b}$ for $I=45^{\circ}$, and in figures $9 \mathrm{a}$ and $9 \mathrm{~b}$ for $I=84.3^{\circ}$. It is rather interesting to see that these are not symmetrical about the north-south geomagnetic axis. It is also evident that the nonreciprocity in the phase velocity becomes greater at the lower frequencies. It is noticed that $Q$ is never more than 4-percent greater than unity. This corresponds to a change of phase velocity of only a few parts in $10^{5}$. 


\section{Concluding Remarks}

In this paper no direct reference has been made to experimental data. In another paper [15] the experimental data on attenuation rates at VLF in daytime [16] were compared with calculations using formulas from the present paper. The agreement between theory and experiment was quite good and the nature of the nonreciprocity between east-west and west-east propagation was satisfactorily accounted for. Unfortunately, the experimental data on phase velocity is quite sparse. It would be interesting to compare the computed results given in this paper with absolute measurements of phase velocities at various frequencies over a given path.

The author thanks Kenneth Spies for carrying out the calculations and Mrs. Eileen Brackett for her assistance in preparing the manuscript.

\section{References}

[1] K. G. Budden, The propagation of a radio-atmospheric, Phil. Mag. 43, 1179 (1952).

[2] K. G. Budden, The "wave-guide mode" theory of the propagation of VLF radio waves, Proc. IRE 45, 772 (1957).

[3] I. A. Al'pert, The field of long and very long radio waves over the earth under actual conditions, Radiotekh. i Elektron. 1, 281 (1956).

[4] W. O. Schumann, Über die Oberfelder bei der Ausbreitung langer, elektrischer Wellen im System ErdeLuft-Ionosphäre und 2 Anwendungen, $Z$. angew. Phys. 6, 34 (1954).

[5] J. R. Wait, The mode theory of VLF ionospheric propagation for finite ground conductivity, Proc. IRE 45, 760 (1957).

[6] J. R. Wait, Terrestrial propagation of VLF radio waves, a theoretical investigation, J. Research NBS 64D, 153 (1960). (Note the inequality at the middle of p. 172 should read $(k a / 2)^{1 / 3} C>>1$.)

[7] B. van der Pol and H. Bremmer, The diffraction of electromagnetic waves from an electrical point source round a finitely conducting sphere, Phil. Mag., \%, 24, 141 (1937).

[8] V. A. Fock, Diffraction of radio waves around the earth's surface, J. Phys. U.S.S.R. 9, 256 (1945).

[9] H. G. Booker, Some general properties of the formulae of the magneto-ionic theory, Proc. Roy. Soc. (London) A14\%, 352 (1934).

[10] J. M. Ratcliffe, Magneto-ionic theory (Cambridge University Press, New York, N.Y., 1959).

[11] N. F. Barber and D. D. Crombie, VLF reflections from the ionosphere in the presence of a transverse magnetic field, J. Atmospheric and Terrest. Phys. 16, 37 (1959).

[12] H. Bremmer, Terrestrial radio waves (Elsevier Publishing Co., New York, N.Y., 1949)

[13] J. R. Johler and L. Walters, On the theory of reflection of LF and VLF radio waves from the ionosphere, J. Research NBS 64D, 269 (1960).

[14] J. R. Johler, private communication (1960).

[15] J. R. Wait and K. Spies, Influence of earth curvature and the terrestrial magnetic field on VLF propagation, J. Geophys. Research 65, 2325 (1960).

[16] W. L. Taylor, Daytime attenuation rates in the VLF band using atmospherics, J. Research NBS 64D, 349 (1960).

\subsection{Additional Reference}

D. D. Crombie, Differences between the east-west and westeast propagation of VLF signals over long distances, J. Atmospheric and Terrest. Phys. 12, 110 (1958).
10.2. List of Symbols

$(r, \theta, \phi)=$ spherical coordinates of observer, $a=$ radius of the earth,

$h=$ height of reflecting layer,

$k=2 \pi /$ wavelength,

$\theta$ is central angle,

$x=(k a / 2)^{1 / 3} \theta$,

$y=(2 / k a)^{1 / 3}(r-a)$,

$i q=(k a / 2)^{1 / 3} \Delta$

$\Delta=\left(\frac{i \epsilon_{0} \omega}{\sigma+i \epsilon \omega}\right)^{1 / 2}\left[1-\frac{i \epsilon_{0} \omega}{\sigma+i \epsilon \omega}\right]^{1 / 2}$,

$w_{1}(t)$ is Airy function of first type defined by eq (4), $w_{2}(t)$ is Airy function of second type defined by eq (6),

$t$ is argument of Airy function,

$\Delta_{i}=Z / \eta_{0}$,

$\eta_{0} \cong 120 \pi$

$Z$ is surface impedance of ionospheric reflecting layer at $r=a+h$,

$i q_{i}=(k a / 2)^{1 / 3} \Delta_{i}$

$y_{0}=(2 / k a)^{1 / 3} k h$,

$w(t)$ is some linear combination of $w_{1}(t)$ and $w_{2}(t)$,

$A(t)$ is defined by eq (8); it is related to the ionospheric reflection coefficient,

$B(t)$ is defined by eq (18); it is related to the groundreflection coefficient,

$C=(2 / k a)^{1 / 3}(-t)^{1 / 2} ; C$ may be geometrically interpreted as the cosine of the angle of incidence at the ground,

$C^{\prime}=\left(C^{2}+2 h / a\right)^{1 / 2} ; C^{\prime}$ may be geometrically interpreted as the cosine of the angle of incidence at the ionosphere,

$R_{\boldsymbol{g}}$ and $R_{i}$ are reflection coefficients at the ground and the ionosphere, respectively,

$\hat{I}=\frac{2 k a}{3}\left[\left(C^{\prime}\right)^{3}-C^{3}\right]$,

$V$ is a dimensionless quantity proportional to the vertical electric field,

$V_{i}$ is defined by eq (13); it may be interpreted loosely as the $\mathrm{j}$ 'th order skywave,

$t_{n}$ is the n'th root of eq (16); it determines the attenuation and phase of the n'th mode,

$\rho=a \theta$; it is the great circle distance between source and observer,

$\delta_{n}$ is defined by eq (32); it is a coefficient in the mode sum,

$F_{g}$ is defined by eq (34); it is a wave correction to the ground-reflection coefficient, $\omega_{r}=\omega_{0}^{2} / \nu$,

$\omega_{0}$ is the (angular) plasma frequency,

$\nu$ is the collision frequency,

$\beta$ is defined by eq (39); it characterizes the ionospheric reflection coefficient $\|R\|$,

$\left[R_{i}\right]=\left[\begin{array}{c}\|R\| \| R_{\perp} \\ \perp R \| \perp R_{\perp}\end{array}\right]$; this is a matrix which describes reflection from an anisotropic layer,

$\mu$ is defined by eq (43); it is a double-valued index of refraction for the ionosphere subject to the $Q L$ approximation,

$\mu_{0}$ and $\mu_{e}$ are the two components of $\mu$,

$\omega_{L}$ is the longitudinal component of the gyrofrequency,

$\omega_{T}$ is the transverse component of the gyrofrequency,

$B=L / H=\frac{2 \pi c}{\omega_{r} h}$,

$H=h / \lambda$

$L=\frac{\omega}{\omega_{r}}=\frac{\omega\left(\nu^{2}+\omega_{L}^{2}\right)^{1 / 2}}{\omega_{0}^{2}}$,

$\phi_{\mathrm{L}}=\tan ^{-1}\left(\omega_{\mathrm{L}} / \nu\right)$,

$P$ and $Q$ are defined by eqs (48) and (49),

$I=$ dip angle of the earth's magnetic field,

$\phi_{a}$ is the azimuthal direction of propagation of the wave; $\phi_{a}=0$ corresponds to propagation from south-to-north, $\phi_{a}=90^{\circ}$ corresponds to propagation from west-to-east, etc.

(Paper 65D1-101) 